\title{
L'altività sismica nella zona delle Sorgenti del Peschiera
}

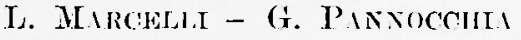

Ricovito il 13 Maggio 1963

\section{I: PARTH:}

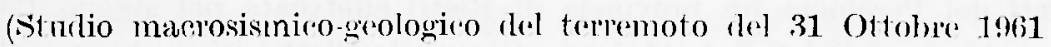

(" pre-istoria sismi(a).

Russuxto. - Questa nota costituisce la Il parte di uno studio dettagliato sull'attività sismiea della zona delle Sorgenti del Peschiera. Ivi vengono analizzati gli aspetti macrosismici del terremoto del 31 ottohre 1961 con epicentro a pochi $\mathrm{km}$ dalle Sorgenti stesse; ai rilievi macrosismici vengono inoltre associati quelli geologici.

i: altresi stata traceiata lit storia sismiea della zona a partire dall'inizo dell'era volgare: numerose carte macrosismiche illustrano i casi più interessanti.

Fssendo stata messa in luee ma certa tendenza della regione a manifestare la sua attiviti sotto forma di "periodi sismici", vengono studiati in maniera accurata alemin tra i periodi piu completi: sono stati messi in grafice gli aspetti più interessanti dei fenomeni (intensità delle singole scosse secondo la scala Mercalli-Sieberg: energie singole e complessive di ciascun periodo; calcolo di pereentuali delle energie associate alle tre fasi fondamentali di ogni periodo).

Lal talbellia A riassume numerieamente i risultati di questi ult imi calcoli.

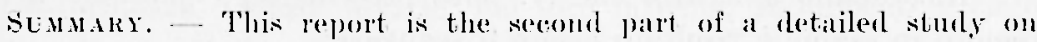
seismical activity in the area of the sources of the river Peschiera. The macroseismical aspects of the earthquake of Oct. 31. 1961. With focus a few kilometres away from the sources itself. ale analyzed. Horeover. geologieal information is added to the macroseismieal data. 
The seismical history of the area, back to the beerimning of the Christian era, is reported. Several macroseismical maps illustrate the most interesting cases.

Since a trencl has been remarked in the area to arrange activity into "seismical periods", some of the most complete periods are carefully surveyed. Craphs report the most interesting aspects of the various phenomena (intensity aceording to the Merealli-sieberg scale; single and total energies of each period: percentage calculations of the energies associated to each period's three fundamental phases.

Table $A$ summarizes in figures the results of the latter caleulations.

\section{RichiaMi.}

In una precedente nota, cui fa seguito la presente, è stato detto come la installazione di una stazione sismica provrisoria presso le Sorgenti del Peschiera (in provincia di Rieti) ellettuata nel giugno 1961 su richiesta dell'A.C.E.A. (Azienda Comumale Elettricità e Acque) allo scopo di studiare la sismicità della zona, abbia messo in luce una attività sismira locale rivelata fin dalle prime registrazioni (primi di giugno 1961). Quando il 31 ottobre dello stesso anno una forte scossa danneggriò gravemente varie località circostanti la zona del Peschiera la stazione funzionava da vari mesi, e poiché ha continuato a fornire regolari registrazioni anche per i mesi successivi (essa è in piena efficienza anche al momento attuale) è stato possibile seguire con particolare attenzione l'intera evoluzione del fenomeno sismico che si ̀̀ presentato come un lungo periodo di attività locale.

Nella precedente nota è stata fissata l'attenzione sulla scossa del 31 ottobre (la più violenta del periodo) ed in uno studio preliminare sono stati dedotti i dati ipocentrali, i valori di $K$, delle velocità delle onde $P g$ ed $S g$, delle prevalenti frequenze di vibrazione del suolo, la magnitudo e l'energia del sismal.

L'intendimento di questa seconda parte è quello di mettere a fuoco l'aspetto macrosismico del terremoto più violento in correlazione con la geologia della zona epicentrale, e di inquadrare il fenomeno particolare in un panorama più esteso nello spazio e nel tempo.

Ricordiamo anzitutto che (v. nota precedente) l'epicentro del terremoto in esame è risultato essere il seguente:

$$
\begin{aligned}
& \varphi=42^{\circ} 21^{\prime} 20^{\prime \prime}, 4 \mathrm{~N} \\
& \mid \lambda=13^{\circ} 01^{\prime} 05^{\prime \prime}, 55 \mathrm{E} \mathrm{Gr} .
\end{aligned}
$$


con nula profondità ipocentrale compresal fra i 3 e i $6 \mathrm{~km}$. Il tempo origrine i

$$
H=13^{\mathrm{n}} 37^{\mathrm{m}} 17^{\mathrm{s}, 6} \text { (G.C.T.) }
$$

del 31 ottobre 1961.

La magnitudo di questo sisma calcolata con le regristrazioni di Roma ì 5,18 .

\section{NoTTZIF M.ICROSISMLCHF.}

Quando ci siamo accinti allo studio di questo terremoto abbiamo cercato di lanecogliere la magrgior quantità di materiale possibile. Si ì già detto che abbiamo potuto disporre di molte notizie macrosismiche. fornite in parte dall'Ufficio Centrale di Meteorologia, in parte selezionate, con un minuzioso ed accurato vaglio, dalle notizie di stampa, ed in parte raccolte con sopraluoghi diretti nelle località più colpite.

E stato così possibile fare le seguenti valutazioni: a Borgo Velino, Antrodoco, Colle Rinaldo, Ponte Alto e Sorgenti del Peschiera si sono verificati danni notevoli. A Borgo Velino molte ease sono state lesionate, sono crollati i merli della torre comunale ed alcuni soffitti in più case, rese perciò inabitabili: lesioni anche al campanile della chiesa. Ad Antrodoco il $4,4 \%$ circa delle case sono state dichiarate inabitabili dal Genio Civile; lesioni ai comicioni; lungro la statale da Borgo Velino ad Antrodoco (ad $1 \mathrm{~km}$ circa da Borgo Velino) si è verificato il crollo di un tratto di circa $10 \mathrm{~m}$ di una spalletta di delimitazione del tracciato stralale; a Colle Rinaldo si sono arute alcune case lesionate ed altre rese inabitabili; a Ponte Alto due case crollate ed altre dichiarate inabitabili: alle falde del Monte Nuria, presso le Sorgenti del Peschiera, caduta di alcuni massi: fra Colle Rinaldo e Rocea di Fondi è stata notata una spaceatura sulla montagna, parallelamente alla strada statale, lunga circa $150 \mathrm{~m}$, profonda dai 30 ai $50 \mathrm{~cm}$, larga altertanto: alle Sorgenti del Peschicun, entro le camalizzazioni di raccolta delle arque dell'impianto A.C.F.A., il tlusso dell'acqua è diminuito di circa. 50 lit ri/sec., e contemporaneamente, lungo il letto del fiume, le acque si sono intorbidate. Queste notizie, nel loro insieme, conducono a circoscrivere l'area di massima intensità macrosismica (grado VIII circa della scala Mercalli-Sicberg) ron una isosista rachiudente le località nominate. Questa isosista presenta una forma allungata pressoché ellittica (v. fig. 1): l'asse maggiore ì nettamente orientato parallelamente 
al corso del fiume Velino, per lo meno relativamente al tratto compreso tra Antrodoco a le Sorgenti del Peschiera. Ijestremo sud dell'asse passa per Pendenza, cosieché lepientro micensismicen si trova in prossimita dellincontro di tale asse con la isosista stessa di grado massimo. Notiamo a questo punto ehe lepicentro macerosismico risulta spostato di qualche km da quello microsismico in direzione NE, ma ciò non ha particolare rilevanza in quanto le manifestazioni macrosismiche sono strettamente legate alla natura del sottosuolo; nel caso partienare la zona ove risulta localizzato leepientro microsismico è pooco abitata, quindi mancano quelle informazioni dirette che inveres si sono potute raceogliere altrove.

Ia isosista successiva alla principale (quella di grado VII) compurende le seguenti località:

Patemo, Cotilia, Castel S. Angelo, Cancera ad oceidente delleppicentro e verso Est Rocea di Fondi; landamento di questa curva segue quello della precedente. Al confine si trovano le localita di Cittaducale, Micigliano, Borbona. Mancano notizie dettagliate della zona immediatamente ad Est a Nord-Est dellepirentro perché poco abitata (Monte Nuria, Monte Nurietta, Monte Morrone, Monte Giano), ma il romportamento della isosista suceessiva (quella di VI) di autorizzal a mantemere il carattere pressoche ellitico delle curve più prossime alla zona epicentrale. Lisosista di VI (r. fig. 2) comprende le località di Petrella Salto, Concerviano, Belmonte in Sabina, Posticeiola, Col di Tora, Rocea Sinibalda, Montereale, Marana e attraversa Borgo S. Pietro e Fiamignano. Eे visibile dal traceiato un andamento simile alle due precedenti isosiste, specie per quanto rigualda la parte superiore della corva (lungo l'asse NE) mentre nella parte inferiore essa curva presenta un allungamento verso Sud-Orest quasi a preanmunciare la preferenza di propagazione del moto sismico, specialmente per le intensita più basse, in tale direzione.

L'isosista di V (Paganica, Monteleone Sabino, Casaprota, Maglianello, Rieti, Capitignano, Barete. Pizzoli) i ancola piuttosto regolare a concorde con quelle di grado più elevato: anomalie notevoli si riscontrano invere per le suecessive. E rimareherole il fatto che mentre in direzione Sud-Ovest (Roma-Rocea di Papa) a $70 \mathrm{~km}$ dall'epicentro l’intensità del fenomeno è stata valutabile al $\Gamma \mathrm{V}$ della scala Merealli-Sieberge, a Nord-Est, già ad una distanza di $38 \mathrm{~km}$ cirea, l'intensità è stata ralutata al II crado della stessa scala (Amatrice).

Questo fatto si ritiene possa essere ascritto alla natura geologica del sottosiolo. 


\section{RH.HEI MACROSISMICO-GEOLOGICI.}

Esaminando la carta geologima al 100.000 v. fig. 3 (foglio 139 del Survizio Geologico) (1) relativa alla zona epicentrale si possono fare degli interessanti rilievi circa la posizione dell'epicentro, cosi come e stata desunta dall'indagine eseguita. Il particolare della zona epicentrale (fig. 1) è stato effettuato su scala al 100.000 allo scopo di rendere più evidenti le eventuali correlazioni tra elementi macrosismici e rilievi geologiei. Fa cosi notato anzitutto ahe le Sorgenti del Peschiera si trovano proprio alla confluenza di un sistema di faglie, una delle quali, orientata verso $\mathrm{S}-\mathrm{E}$, passa per Pendenza e prosegue oltre arrestandosi all'imboccatura di una dolina; questa faglia sembra a questo punto proseguire spostandosi di circa un centinaio di m verso sud, poi, aggirando la dolina stessa, prosegne verso E-NE in direzione di Serra Secca. Da Pendenza un'altra faglia si mantiene a nord delle precedenti, e all'altezza della dolina prosegue palallelamente anch'essa in direzione di Serra Secea. L'epicentro, vieinissimo a Pendenza, sembra vada localto in questa zona fagliata. In questa zona che si estende a sud-est del Velino, per un'area rettangolare di circa 15 kmq predomina il cretaceo superiore. Questo rettangolo, verso S-E è a contatto tettonico per faglia con il complesso dei Monti Nuria e Surietta (del cretaceo medio), mentre gli altri tre lati confinano con delle strutture notevolmente eterogence in cui sono sensibili vaste porzioni di miocene con qualche isola di terereni riferibili probabilmente al pliocene, estensioni noteroli di quaternario di tutte le etì, dal pis antico pleistocene al piì recente olorene, in cui scorre il Velino.

In questa zona om descrita è stata tracciata la isosista di massima intensiti. Ad Antrodoco, che poggia su ma formazione attribuita al trias la quale fa parte di nua catena mesozoica (con direzione NWSE) che chincle a Nord la concal alluvionale del Velino, i danni sono stati notevoli come si è visto (valutati trá l'VIIT a il VII grado dellar seala Mer(alli-Sieberg): e cosi pure a Borgo Velino, Ponte, Canetra, Cotilia, Paterno, dislocate ai bordi di questal conce, lungo il corso del fiume stesso, per quanto nelle ultime tre localita -- Canetra, Cotilia, Paterno

- l'intensità del sisma sia giunta lievemente smorzata (di VII) rispetto

(1) Per motivi tipografied la carta viene qui riprodottain bianco e nero. Per una nighiore comprensione della deserizione che segue, consigliano però l'osservazione sulloriginale a colori, edito dal Servizio fieologico. 
alle altre; la isosista sembra poi aggirare le Sorgenti del Peschiem, Pendenza e, passando per Roceal di Fondi, richiudersi verso Antrodoco.

Ia presenza di numerose doline sparse nella zona epicentrale, sperrialmente nel rettangolo fagliato sopla descritto, tra il Velino d il Monte Suria, denuncia una struttuma carsica sottostante. A questo proposito ricordiamo she i valori che nella prima parte di questa ricerea sono stati trovati per le velocità di propagazione delle onde Pg ed sg si arvicinano molto a quelli trovati da altri autori per zone carsiche: questo fatto confermerebbe le ipotesi aranzate.

E interessante altresì porre in eridenza qualche altro elemento di raffronto.

Castel S. Angelo, Cotilia, Canctra e Paterno, dove la scessa ò stata sentita di VII, si trovano ai bordi di un deposito di olocene, mentre Rocea di Fondi si trova in prossimita di una faglia, soppra uno straterello di paleogene ronfinante con una larga porzione di restaceo superiore.

Borbona e Micigliano, che si trovano ai confini clella isosista di VII, poggiano entrambe su una formazione marnosa molassica del miocence, mentre Cittaducale si trova sul quatemario più antico.

Una menzione a parte meritano le due località di Santa Rufina ( Casperia. In entrambe la scossa è stata valutata di IV grado, mentre Santa Rufina si trova entro la isosista di V a Casperia addirittura entro la isosista di HI. Dall'esame della carta geologica al $1.000 .000 \mathrm{sem-}$ bra che le due località si trovino su ma nguale formazione geological.

Va notato altresi, senza tuttavia pretendere di dare una interpretazione al fenomeno, che le zone di magroviore deformazione delle isosiste di grado più basso sono due (v. fig. 2): una in direzione Sud-Est rispetto all'epicentro (ed in tale direzione prevale nua formazione geologica del mesozoico sopracretaceo $-\mathrm{C}_{2}-$ ) ed una ad essa pressoché ortogonale, in direzione Sud-Ovest. In questa direzione l'ossatura ò ancoma del mesozoico ricoperto da seclimenti più recenti che arrivano fino al quaternario ivi comprese lo vulcaniti appartenenti al Vulcano Laziale.

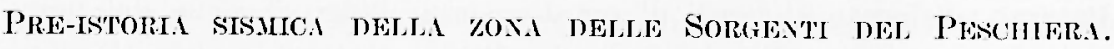

i noto ed eridente che una manifestazione sismira non costituisce mai un fenomeno a se stante, isolato nel tempo e nello spazio. Essa è l'aspetto più clamoroso di uno stato di tensioni a di equilibri instabili cui isottoposta la terra nella sua totalita. Zone di minor resistenza della crosta terestre sono ovviamente quelle che piǹ di frequente sono teatro 
di convulsioni telluriche, o manifestazioni volcaniche. È altresi evidente che il raggiungimento di ma posizione finale di equilibrio di ma determinata zona può forse arverarsi in lassi di tempo dell'ordine delle ere geologiche, ed essendo ancora sconoscinte le cause remote dei terremoti. non si può a maggior ragione, spingersi nel futuro con previsioni o ... profezie. Si può però guardare indietro nella storia sismicua di una regione allo scopo di inquadrare il fenomeno attuale in un panorama più esteso di cui esso è entrato a far parte e questo non per trarne delle deduzioni né tanto meno permettersi delle induzioni, quanto per portare un modesto contributo alle conoscenze fin ora acquisite.

A tale scopo abbiamo consultato le documentazioni relative alla sismicità dell'Italia a partire dall'inizio dell'era volgare e ne abbiamo tratto tutte quelle notizie che abbiamo ritenuto utili al nostro scopo. Tali documentazioni, ovviamente piuttosto sommarie nelle epoche più antiche, si sono andate via via arricchendo col passare dei secoli, tanto da permetterei di costruire, per moltissimi casi, delle carte macrosismiche abbastanza complete e significative.

Saturalmente, nella nostra ricerea, abbiamo selezionato soltanto quei fenomeni che hamno interessato direttamente o da vicino la nostra zona epicentrale. Abbiano ritenuto opportuno considerare un'area di raggio circa $50 \mathrm{~km}$ intorno alle Sorgenti del Peschiera in modo da includere quella porzione della dorsale appenninica cui appartiene anche il massiccio del Gran Sasso.

I terremoti con epicentri un po' più lontani (ma non oltre gli 80 $\mathrm{lim}$ ) sono stati inclusi nell'elenco quando hamno avuto delle ripereussioni di una certa importanza; dalle figg. 5-6-10-16-18 per esempio si rede infatti che la rete delle isosiste copre la nostra zona anche con gradi alquanto elevati. Riteniamo lecito supporre che $\mathrm{i}$ focolai sismici di tutta la regione presa in esame, siano collegati gli uni agli altri si da formare un complesso unico: forse da questo panorama di insieme vammo esclusi i centri di Cascia e Norcia (Cascia in particolare) la cui attività si manifesta di solito con delle caratteristiche tutte proprie. Siccome però in alcuni casi (sett. 1349, ott. 1599-gemn. 1600, gennaio-febb. 1703 . giug. 1719, febb. 1879) si è avuta notizia di estese ripercussioni macrosismiche, non abbiamo ritenuto opportuno sottrarre questi elementi ad un quadro di insieme, pur ritenendo doveroso sottolineare l'aspetto caratteristico dell'andamento sismico di Cascia che, specie per un'attività moderata fa, come si è detto, di Cascia, un centro tutto indipendente.

Sottolineiamo inoltere il fatto che l'esame macrosismico cosi esteso ('i consente altresi di avere una indicazione della sismicità anche di 
quella porzione delle regioni Cmbria e Tazio lungo cui si snoda l'acquedotto del Peschiem.

Vediano così clue le notizie storiche più antiche risalgono:

(1) * all'anno 678 di Roma; in quest'anno Ricti fu colpita da un terre$I_{0}=V I I I$ moto rovinoso (VIU). Ne dà notizia, in una cronaca $A \cong 1+\mathrm{km}$ latina il "Dei Prodigii" _. l'autore G. Obsequente.

*? Il 1216 p. C., a Subiaco (Roma) un terremoto rovinò del tutto il $I_{0}=$ VIII Monastero di S. Scolastica.

$1 \cong 48 \mathrm{~km}$ Naturalmente mancano notizie piì particolareggiate ma trattandosi di un VIII grado della Seala MercalliSieberg ì da presumere che esso abbia aruto estese riperenssioni.

*:**** Nell'anno 1246 si ha notizia che a spoleto "frequenti e fortissimi $I_{o}=$ VII terremoti scossero talmente la città che fecero diroccare $1 \cong 45 \mathrm{~km}$ molte case e molte torrin.

'Tali scosse, eni si può attribuire un VII grado (per lo meno per le più forti), durarono tutto l'anno.

Si tratta dunque di un periodo sismico che ha interessato la zona di Spoleto. Osserviamo a questo punto che riteniamo molto interessante far rilevare il fatto che la sismicità dei vari centri distribuiti qua e là nell'area che abbiamo preso in esame, si presenta per lo più sotto l'aspetto di periodi sismici, più o meno lunghi, più o meno intensi, come avremo occasione di redere nella esposizione che segue. Rari sono i casi di scosse isolate o che si esauriscono rapidamente.

(1) Indichiamo con:

$I_{0}$ la massima intensità epicentrale;

A la distanza approsinimativa di ciasenua zona epiecntrale dalle sorgenti del Peschiera;

$I_{P}$ la intensitì con cui la seossa è stata sentita alle Sorgenti del Pescheliera (quando è stato possibile dedurla).

Inoltre indicheremo con:

*k** $\mathrm{i}$ terremoti appartenenti ad 111 periodo sismico pinttosto lungo;

*** i terremoti appartenenti an $1 \mathrm{~m}$ periodo sismico breve;

** pochissime scosse ron lo stesso epicentro (non più di 3 o 4 );

* scossia isolata. 
Mat su tale argomento, che merita particolare attenzione, tomeremo più avanti. Per ora ci linitiamo a segnalare il fatto.

*k:k:* Ancora mellanno 127\% a Spoleto "tervibili scosse fecero rovinare $I_{0}=$ VIII molti edifici con mortalitic di persone".

$\Delta \cong 45 \mathrm{~km}$ Anche qui si tratta di un periodo sismieo le cui seosse più forti raggiunsero l'VIII.

*k:*: Il 30 nov. 1298 a licti e spoleto crollarono (ase a palazzi. Nume$I_{0}=$ VIII-VII rose furono le vittime. Le scosse più forti furono di $\Delta_{1} \cong 15 \mathrm{~km} \quad$ VII-VIII.

$A_{2} \cong 45 \mathrm{~km} \quad$ (Fu un periodo sismico dulato $6 \mathrm{~m}$ mesi).

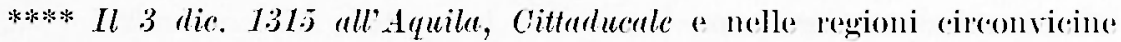
$I_{0}=$ VIII avvenmero dai terremoti rovinosi (VIII).

$\Delta_{1} \cong 20-25 \mathrm{~km}$ ? Fortissime repliche si susseguirono per 30 giomi. Non $A_{2} \cong 6 \mathrm{~km} \quad$ i da eschulere che anche a seguito di queste "fortissime "repliche continuasse ma attività più modesta e per una maggiole durata, che la cronaca del tempo, preocoupata dei fenomeni più clamorosi, non hat messo in riliero. Ha anche se eflettivamente le repliche si fossero ripetule solo per 30 giorni, si tratterebbe pur sempre, anche qui, eli un periodo sismico.

*:*k:* Il 9 o 10 settembre 1349, L'Aquila, il sannio e tutti gli Abruz i $I_{0}=\mathrm{XI}-\mathrm{X}$ subirono una immane catastrofe che per intensità ed $A \cong 35-40 \mathrm{~km}$ estensione si può paragonate a quello che nel 1703 sconvolse letteralmente le stesse regioni, come redremo piò avanti.

Dalle notizie raccolte per questo violentissimo terremoto si può desumere che allepicentro esso abbia raggiunto il X-XI della Seala Mercalli-Sieberg. Lepicentro stesso non fu facilmente ubicabile, tuttavia si sa che I'Aquila fu quasi completamente distrutta: 800 furono ivi i morti. A Montecassino "crollo dallo fondamenta il Momastero facombo memerose rittimen. S. Germano fu per metà distrutto. Ancora distruzioni imponenti a numerosissine vittime si ebbero a Sola, Atina, Venafro, Campobasso, Isemia, Arersa, Xapoli, s. Agata in Puglia. Anche a Roma ci furono 
rolli e gravi damni, e cosi pure a Terni, Perugia, San Sepolcro, Assisi, Spello, Spoleto, Orvieto, Viterbo. Questo terribile sconvolgimento fa parte esso pure di un lungo periodo sismico che inizia probabilmente fin dal gennaio 1349. Ad esso seguirono poi numerose repliche delle quali però non si hamno notizie dettagliate.

Poro più di un secolo dopo, il

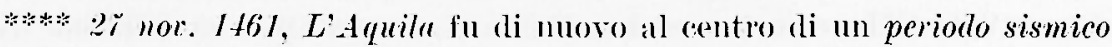
$I_{0}=$ IX-VIII durato più di due mesi. Dell'intero periodo si hanno $1 \simeq 35-40 \mathrm{~km}$ notizie un po pii particolareggiate delle preceslenti, tanto che possiamo dare un elenco delle scosse piu forti, con le relative intensiti.

Il periodo sembra iniziare il

J6 noe. $1+61$ ron mal scossa di IV-T; a distanza di 11 griorni, il

27 now alle ore o5 circa si ebbe la scossa più violental di tutto il periodo: essa si puó valutare di VIII-LX. All'Aquila si ebbero distruzioni e molte vittime, e cosi pure a S. Eusonio, Castelnuowo, Onda, Poggio Picense, Teramo. A Perugia e Roma fu sentito di IV.V. Probabilmente anche Cittaducale ebbe a soffrire qualehe damno. Dopo questa, seguirono numerose repliche: una il

t die. - di VI, joi un rentinatio di minori fino al

I.j dic. - poi il

It dic. (ore os rirea) una di VI-TII

t genn. It6: fore 04 cilea) una di V.YI

t yenn. (ore og rirca) una di VI a poi alte minori fino al $\because$ febl. $1+6 \%$.

*k* /I aprile It!gs - L'Aquila subì di nuovo una scossa di VII, e il

$I_{0}=$ VII

$1 \simeq 30 \mathrm{~km}$

I: "uprile 1498 - ancoma a $L$ Aquilu un'altra della stessa intensità (VII) $I_{0}=\mathrm{VII}$

$1 \simeq 30 \mathrm{~km}$ 
**an: Nellammo 1.j02 - Cittalueale fu teatro ancliessa di un periodo $I_{0}=$ VLII sismico: le scosse, alcune delle quali rovinose, conti$1 \cong 6 \mathrm{kmll}$ muarono per 40 giomi. Si hal notizia di molti arolli e lesioni in numerosi colifici: le accpue delle fontane si intorbidarono.

La mancanza di notizie piu particolareggiate non consente la costruzione delle isosiste. Si puo ritenere tuttavia che alleppicentro le scosse piu forti abbiano l'aggiuunto l'VIII.

* Il :0-21 aprile 1:jy 1 a Spoleto si ebba . . "un terremoto si viotento che $I_{n}=$ VI-VII pareva dovessero subissare gli selifici" (Pensiamo poter $.1 \cong 45 \mathrm{~km} \quad$ valutare tale sisma di VI-VII).

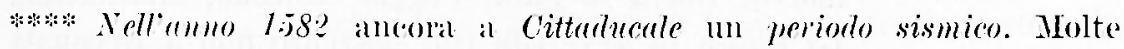
$1 \supseteq 6 \mathrm{~km} \quad$ scosse durinte tutio l'anno.

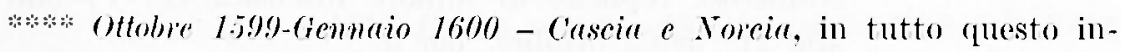
$I_{0}=\mathrm{IX} \quad$ tervallo di tempo furomo battute da numerosi e vio$1 \simeq 4 \%$ lin lenti terremoti.

Il periodo sismico hal inizio fin

dallottobre l:599 con delle scosse lievi (di III-IV). Poi il t nov. si ebbe un teremoto di VI, cui fece seguito il

j nor. (ore 0.5 (ircal) la più violentar a rovinosa scossa della serie (LX alleppice). Cascia, Sorcia e circondario chbero a subire vittime e crolli. La perecezione macrosismicar di questo sisma giumse fino ar Roma, Perugial. LiAquila, Aneona, e Pessitro.

Forse le scosse violente furono più d'una: le notizie, pur parlando al plurale per quanto riguarda ele scosse più violente n damno una visione globale degli effetti macrosismici. Sono tuttavia specificate, fino al

6) gemmaio 1600, molte repliche forti (ali V): da questo griormo si fecero piu rare a deboli (III-TY).

1110 genmaio l600 una nuoral scossat intensal quasi come le prime (VI).

*? Nel 1616 con Spoleto come probabile epientro un terremoto di VI $I_{n}=\mathrm{VI} \quad$ rireal fu sentito in tuta la Sabina e in tuta l'Lmbria. $1 \simeq 4.5 \mathrm{~km}$ 
**a:* L'8 oflobre 16.39 ald Amatrice inizia un periodo sismico durato $I_{0}=$ VIJI-1X cirea 10 giomi. Una prima scossa si ebbe il

$1 \cong 35 \mathrm{~km} \quad 8$ offobre I6339 alle 07" di $\mathrm{V}$; una seconda, più forte, il

8 ottobre 16:39 alle or" 15$)^{\mathrm{m}}$ di VI; o poi la più violenta, sempre il

Sollobre 1639) alle 07" $30^{\mathrm{m}}$ di VIII-LX. Per questo sismal numerose localitì, oltre Amatriee, furono sconvolte, con morti, foriti, crolli e rovine. Le piì eolpite furono: Campotosto, s. Martino, Collalto, Pinaco, Capricchia, corva, Forcella, Filetta, Nescalia, Pedarga, Cantone, Colle Raso, Corsenito, Casale, La Rocal, Tormetta, Pasciano, S. Jorio e c'ollemoresco, Acemmoli (nnolti morti), Rocoal di Salli, Poggrio Cancello, Montereale. La scossa fu arvertita con spavento fino a Recanati e a Rieti, però senza danni.

Numerose repliche di minore intensita $(\mathrm{IV}-\mathrm{V})$ fino alle 21" dell's ottobre, poi il

It ottobre 16:39) (alle in $30 \mathrm{~m}$ ) ma replical molto violenta (VII-VIII) a un'altral fortissima il

Ir ollobre 1639 (di VI-VII). Le notizie si arrestano al questo terremoto.

***** Vell'aprile 1646 comincia all'Aquile un nuovo periodo sismico.

$I_{\mathrm{o}}=\mathrm{VII}$ Si hat notizia che esso durò fino al giugno dello stesso

$1 \simeq 30 \mathrm{~km}$ anno: si ebbero 166 scosse aleme dello quali di VII.

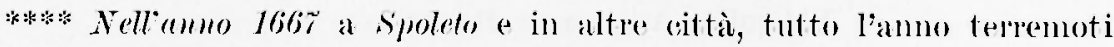
$I_{0}=$ VIII rovinosi. C'i troviano pereio di nuovo di fronte ad $\Delta \cong 45-50 \mathrm{~km}$ un periodo sismico con dello punte di VIII.

* L's giugno 1672 (alle ore 23) Amatrice, Hontereale o T'Aquila subi$I_{0}=$ VIII-VII rono gravi danni per un teremoto di VII-VIII.

$\Lambda \cong 30 \mathrm{~km}$

*:k:k Sel gemmaio-febbraio 1703 alemi tra i più riolenti terremoti che la storia sismiou italiana ricordi scomvolsero i territori di Norcial, Cascia e L'Aquila.

Alcuni fenomeni precursori ermo stati già segnalati nell'ot tobse 1702. Ia seosse catastrofiche furono tre: 
I) 14 genn. 1603 (alle ore 02 ) ron epiecentro tral Iroreia, $I_{0}=\mathrm{X}$-XI Cascin e Leonessar. Si puó ritenere che ivi sia stato $1 \cong 40-45 \mathrm{~km}$ raggiunto il X-XI grado della scalat Merealli-Sieberg. $I_{P} \cong$ VILE Norcia rovino completamente; Cascia fu, dopo Noreia, la città più dammeggeiala.

Il terremoto fu violentemente sentito anche all Aquila, Roma, Foligno. A Roma le accue cambiarono di livello e di colore.

II) $16 \mathrm{genm} .1703$ - ore 21 (X-XI) fu prevalentemente $I_{0}==\mathrm{X}-\mathrm{XI}$ sentita a Montereale (con 80 vittime e distruzione \lrcorner$\cong 25-30 \mathrm{~km}$ quasi completa clella (ittì), Cittareale, Cittadureale, $I_{P}=$ V.III Borbona, Accumoli, Amatrice (distrutte quasi completamente). A Posta a Leonessa gravi danni. Fu arvertita fortemente ad Ascoli a lievemente a Romat.

III) ¿ febl. 1703-ore 18. Questa scossa fu la più forte $I_{0}==$ XI-XII delle tre (XI-XII). Il suo epicentro 2 proprio $l: 1$ $1 \cong 30-35 \mathrm{~km}$ quile la quale fu quasi completamente rovinata: nes$I_{P}=\mathrm{IX}-\mathrm{X} \quad$ sun exlificio restò immume, neanche i più solidi. Ci furono 2500 morti nella città e T.694 nel territorio alquilano.

Numerosissime repliehe con eporeentri localizzati qua e là nella zona paldicolatrmente colpita dalle tre formirlabili scosse, perduralrono per oltre un anno. (Onal delle più forti, quella del J) febbrain colpi in modo particolare Rieti e il rircondario, come vedremo oltre). Una menzione particolare merita cluestal immane (attastrofe. Colla fig. I abbiamo tentato di ricostruire le isosiste in base alle notizie raceolte dal Baratta. In panuoso elenco di localiti completamente lase al suolo, o distrutte, o rovinate completamente o guasi dai mon'idea clella gravita del fenomeno. Migliaia di vittime unatne si regist rabono: nel solo paese di Arendita, presso Cascia, l'81." clegyli ahitanti mori a causa del terremoto; a Paganelli il $73 \%$, a Savelli il $52 \%$ al in tante altre localita dal 40 al $50 \%$. Foreial fu distrutta completamente e Cascia quasi.

Lal scossal del 2 fobbrato sposta il suo epicentero nell'Aquilano, talehé si hanno due reti di isosiste, non 
facilmente distinguibili. Sarebbe stato impossibile farne tre. I dammi delle ultime scosse, come suole arvenire in questi aisi, si sovrappongono ai precedenti e non è possibile separare con certezza i fenomeni. Sta di fatto tuttavia che in occasione di questo periodo convulsivo fu interessata in morlo violento anche la zona immerliatamente circostante le Sorgenti del Peschiera: Pendenza fu "abbatuta interamente ", Antrodoco fu "rovinata dalle fondamenta" e ivi si ebbero 300 morti e 50 feriti, Castel S. Angelo fu "assai danneggiata", a Cittaducale si ebbero " + case cadute $e$ molte lesionate ed inabitabili ", a Rieti si ebbero repliche frequenti per t giomi con danni e rovine agli exlifici... Molto interessante altresì $r i$ è semblato il fatto che in occasione della scossa del 2 febbraio si apri una voragine nei pressi del Monte Omaro, virino a Sigillo. Le docmentazioni dellepoca riportano che "essa era larga camne 20 e lunga 25; con una fune munita di grosso contrappeso, quantunque lunga 300 canne, non si riusci a toccane il fondon. Si racconta che al momento della formazione di tale squarciatma uscirono "campe di fuoco" e che "per altri tre gioni vennero emessi dei vapori".

Ralpportate alle misure odierne le dimensioni di detta " voragine " sarebbero all'incirca le seguenti: larghlezza $45 \mathrm{~m}$, lunghezza $56 \mathrm{~m}$, profondità oltre $670 \mathrm{~m}$. Notiamo a questo proposito che la ubicazione di questa rolagine va posta ad una distanza di appena 15 km dall'epicentro del terremoto ougrgetto del nostro studio, si che quando altrove abbiamo supposto trattarsi di zona carsica, risulterebbe confermato da queste ulteriori docmentazioni.

Periodo Sismico $1 \% 03-$

Abbiamo tentato di ricostruire il periodo sismico cui appartengono questi parossismi. Quasi sicuramente esso a incompleto e inesatto, perché le notizie larcolte qua e lit non sempre si riferiscono alle zone epicentrali talclué ocorrerebbe poter estrapolare le valutazioni macrosismiche disponibili, cosa praticamente impossi- 
bile: inoltre certamente salanno sfuggite alla ricerea di quel tempo numerose repliche di moderata intensità. Comunque già il seguente elenco dà un'idea abbastanza interessante dell'andamento del fenomeno: Fin dal mese di

ottobre 1702 alcune lievi scosse furono avvertite in Unllbria (di III), il

18 ottob. (ore 14 circal) fu segnalato un VI a Norcia, un IV a Roma,

nel mese di novembre frequenti terremoti di cui alcuni di VI-VII a Cascia, il

1t nov. fu segnalato un VII a Foligno (si tratta di una scossia locale e (irroscritta?), il

1t gennaio 1703 (ore 02 circa): X-XI all'epicentro (I delle tre grandi scosse). Il

$1 \pm$ gennaio (ore ?) - VI?

Numerosissime repliche. Il

16 gennaio 1703 (ore 21): X-XI all'epicentro (II delle 3 grandi scosse)

Numerose repliche quotidiane. Il

2.5 gennaio segnalato un V-VI a Norcial. Il

2 febbr. 1\%03 (ore 18) - XI-XII all'epicentro (III grande scoossa). Il

$3 f e b b r$. 1\%03 $\left(20^{11} 45^{m}\right)$ - segnalato un VI a Roma. Dal 3 al 2:5 febbr. segnalate quotidianamente una o più scosse a Roma (di III). Il

1.j febbr. (ore 07 ) - segnalato a Rieti un VII-VIII con repliche per 4 giorni.

$2 j$ febbr. (ore 24) - segnalato un III a Norcia, il

26 febbr. (ore 03) - segnalato un IX a Norcia, il

$26 \mathrm{febbr}$. (ore 04) - segnalato un IV? a Norcia, il

26 febbr. (ore 05) - segnalato un V a Norcia, il

26 febbr. (ore 06) - segnalato un IV? a Norcia, il

$26 \mathrm{febbr}$. (ore 09) - segnalate due scosse di III-IV a Norcia, il

It maro segnalato un $\mathrm{V}$ a Nami, il 
18 marzo segmalate alcume seosse rovinose (di VIII) all'Aquila. il

2l marzo segmalalo m $\mathrm{V}$ a Roma (Albano, Frascati, Castel (randolfo), il

gi maro segnalato un VIII all'Aquila (sentito anche a Roma, Foligno, Spoleto), il

31 maro (ore 12 (irca) segnalate aleune scosse di IV a Roma e I Aquila, il

1 aprile (ore 17,30 (iir(a) segmalato un IV? all'Aquila, il

2 rprile seconalate alcune scosse di IV?; il

$\$$ aprile (ore 12) segmalato $10 n$ VIII a Cascia (sentito an(he a Roma), il

8 aprile (ore $18-19$ circa) segualate alcune scosse a Roma (IV?), il

1.) "prile segualat:a una scossal all'Aquila (IV?), il

$2+$ aprile (ore 21 circa) segmalata ma scossa all'. Iquila (VI?). Sentita anche a Roma, il

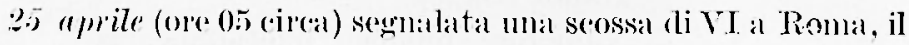
29 giugmo (ore 23) segulalata ma scossal di VII a Spoleto a palesi vicini:

fino a tutto il mese di agosto si sono sentite mumerose repliche avertite fino a Terni:

nell'oftobre fu segmalato un IVo a Nami

nel dicembre altre seosse di VI-VII a Noreia, Narni, Terni, Spoleto, il

$2 \pi$ febbrrio liot ancora un ППГ-TVo arrerdito a Foligno.

A questo tormentalo periodo fammo seguito alcuni anni di quiete. Una importante ripresal si ebbe il

* 27 giugno $1719-\left(10^{\mathrm{H}} 30^{\mathrm{m}}\right)$ quando a Norcia e circondario una scossa $I_{o}=$ VIII di VIII fece crollare alcune ase. Vi furono anche $\Delta \cong 45-50 \mathrm{~km}$ alcuni morti, a la perecezione macrosismica giunse fino a Roma. A Rieti, Spoleto, Foligno ed altue località fu sentita fortemente (di V). 
*? 29 marzo 1776 - Rieti (VI-VII) - Una forte scossa nel territorio di $I_{0}=$ VI-VU Rieti si propagò anche nell'Abruzzo. In alcuni luoghi $\Delta \cong 15 \mathrm{~km}$ causò rovine di case, ma non vittime. (Mancano notizie più particolareggiate).

**** 2 e 9 ottobre 1785 - Piediluco - Comincia nell'ottobre un lungo , $\left[I_{0}\right]_{1}=V W \quad$ ed intenso periodo sismico che dura anche parecehi , $\Delta \cong 28-30 \mathrm{~km} \quad$ mesi dell'anno successivo. Le scosse del 2 e del 9 ot1 $\left[I_{0}\right]_{2}=$ VHI-IX tobre 1785 furono le più violente e furono sentite ab$\Delta \cong 28-30 \mathrm{~km} \quad$ bastanza forti (di V) anche a Rieti e Cittaducale. Il periodo si può riassumere, approssimativamente, nel modo seguente:

2 ottobre $1785\left(3^{\mathrm{h}}+5^{\mathrm{m}}\right)$ - VIII a Piedilueo

Numerose repliche (più di 40) di III-IV per tutto il giorno e la notte successiva

2 ottobre (ore 22 circa) - IV a Piediluco

t ottobre (ore 18 circal) - VI a Piediluco

giorni 5 e 6 ottobre - varie a Piediluco di IV

9 ottobre (ore $0+$ circar) - IV a Piediluco

9 ottobre $\left(04^{\mathrm{h}} 15^{\mathrm{m}}\right.$ circa $)$ - V a Piediluco

9 ottobre $\left(04^{\prime \prime} 30^{m}\right)$ - VI a Piediluco. Sentito anche a Rieti.

9 ottobre (ore 10) - VUI-IX a Piediluco. Fu sentita di V a Rieti e Cittaducale.

13 ottobre $\left(01^{\text {n }} 5^{\text {nn }}\right)$ - IV a Piediluco

13 ottobre (ore 21) - V-VI a Piediluco

Varie repliche (di $\amalg-I V$ ?) fino al 23 ottobre.

5 novembre (al tramonto) una scossa avvertita (di IV?) a Terni.

12 novembre (ore 01) una scossa avvertita ( $\mathrm{TV}$ ?) a Terni

12 novembre (ore 03) una scossa avvertita di $\amalg$ a Roma

Per tutto il mese e nel mese successivo varie altre repliche non identificate fino al

20 dicembre in cui si ha notizia di più di 6 scosse sentite. Ancora in data

11 gennaio 1786 si ha notizia di frequenti scosse sentite a Terni e territori vicini (di IV $-\mathrm{V}$ ). 
febbraio - sentite altre repliche

maggio - parecchie scosse sentite di VI circa a Terni, Narni, erc. ...

21 giugno - 9 scosse a Spoleto (alcune di VII)

26 luglio - (ore 16,30) una scossa di VI sentita a Terni. Varie repliche di minor intensità.

*? 22 marzo 1821 - Rieti - La città fu gravemente danneggiata (man$I_{o}=$ VI-VII? cano altre notizie) (VI-VII?).

$\Delta \cong 15 \mathrm{~km}$

* $2 \bar{j}$ novembre 1842 - Cittaducale - Una scossa di IV.V.

$I_{0}=\mathrm{IV} \cdot \mathrm{V}$

$\Delta \cong 6 \mathrm{~km}$

* 24 settembie 1853 - Cittaducale - Una scossa di $I T-V$.

$I_{0}=\mathrm{IV}-\mathrm{V}$

$\Delta \cong 6 \mathrm{~km}$

* 10 marzo 1850 - Cittuducale - Una scossa di IV-V.

$I_{o}=\mathrm{IV}-\mathrm{V}$

$\Delta \cong 6 \mathrm{~km}$

**** 15 settembre $18 \% 8-\left(8^{\mathrm{n}} 20^{\mathrm{m}}\right)-$ Umbria VII-VIII presso le Fonti del $I_{0}=$ VIII-VII Clitumno.

$\Delta \cong 60 \mathrm{~km} \quad$ Nlle $8^{\mathrm{h}} 20 \mathrm{~m}$ del 15 settembre ha inizio un periodo $I_{p}=\mathrm{IV}-\mathrm{V}$ sismico che dura per tutto il mese. La zona epicentrale si trova nei pressi delle Fonti del Clitunno. L'VIII-VII grado della scossa principale ha investito le localita di Castel Ritaldi, Montefalco, Bruna, Mercatello, Fratte, Turrita, con crolli e nmmerose rovine. La estensione dell'area macrosismica è piuttosto ampia e dalle isosiste della fig. 5 si vede chiaramente come la zona delle Sorgenti del Peschiera anche in questo caso sia ampiamente contenuta entro i confini di una isosista di grado piuttosto elevato (V-IV).

Frequenti repliche: le più importanti sono:

15 settembre $\left(13^{n} 53^{\mathrm{m}}\right)$ - anche questa di VIII-VII. 
16 settembre $\left(13^{n} 55^{\mathrm{m}}\right)$ - di VIL.

Moltissime repliche tra il V e il VI. Altre più lievi (IV-V?) fino al 19. Ancora più lievi (III-IV?) fino al 25. Poi si abbe mal ripresa il

30 settembre (pomeriggio) [V-VI?] con altre repliche minori.

**** 23 febbraio $18 \% 9\left(19^{\mathrm{n}} 30^{\mathrm{m}}\right)$ - Epicentro: Cascia-Norcia - Prece$I_{0}=$ VIII-VII duto da molte piccole scosse (il 22 e il 23 febbraio $\Delta \cong 40-45 \mathrm{~km} 1879$ ) alle $19^{\mathrm{\prime \prime}} 30^{\mathrm{m}}$ del 23 una forte terremoto (di VII-VIIT) colpi Cascia, Norcia e Serravalle. A Cascia "tutte le case si fendettero da cima a fondo". Ta scossa fu forte (di V?) anche a Roma, Ascoli P., Camerino, Amelia; di IV all'Aquila.

Data l'estensione è cla ritenere che, pur non essendo state nominate specificatamente anche le località di Rieti e Cittaducale esse siano state interessate in maniera sensibile.

Numerose repliche, di cui però mancano notizie dettalchiate.

Ancora una volta di troviamo in presenzil di un periodo sismico.

*\%*\% 11 marzo 1881 - Umbria - Nel quadro di un nuovo intenso periodo $I_{0}=$ VII sismico che interessò moltissime città umbre, particolamente nel mese di marzo 1881 (giorni 11 e 12), ma con strascichi per tutto l'anno, è specificatamente indicata la città di Rieti con $t$ scosse in due ore. Le principali seosse di detto periodo sono:

4 marzo $\left(14^{\mathrm{h}} 10^{\mathrm{m}}\right)$ Sentita di III a Spoleto

\% marzo $\left(02^{n} 30^{m}\right)$ Sentita di III a Spoleto

8 marzo $\left(10^{\mathrm{n}} 46^{\mathrm{m}}\right)$ Sentita di IV a Spoleto

9 marzo (02" (irca) Sentita di III a Spoleto

11 mar $\approx 0\left(11^{n} 15^{m}\right)$ Sentita di IV a Foligno; ali III a Spoloto

11 maro (12" 30m) Sentita di IV a Castel Ritaldi, S. Giovami; di $\amalg$ a Spoleto, Assisi, Perugria, Todi

$1 /$ marzo (16" $\left.55^{m}\right)$ Sentita di VI a Foligno, Trevi, Castel Ritaldi, Campello; di $V$ a Spoleto 
11 marzo (23" $\left.50^{\mathrm{m}}\right)$ Sentita di VI a Foligno, Trevi, Castel Ritaldi, Campello: di $\mathrm{r}$ a Spoleto

11 marzo (...) Varie scosse nella giomata sentite a Rieti

11-12 marzo (di notte) 19 scosse sentite a Terni di cui alcune di VII

11-12 marzo (di notte) 4 scosse sentite a Rieti in due ore. Altre riprese nel mese di novembre.

* It agosto 1881 - Cittaducale (scossa di IV-T).

$I_{0}=\mathrm{IV} \cdot \mathrm{V}$

$\Delta \simeq 6 \mathrm{~km}$

* 18 novembre 1881 - Cittaducale (scossa di IV-V).

$I_{0}=\mathrm{IV}-\mathrm{V}$

$1 \cong 6 \mathrm{~km}$

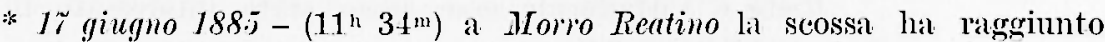
$I_{0}=$ VII $\quad$ il VII; a Rivodutri il VI: a Rirti, Leonessa e Terni $A \cong 25 \mathrm{~km} \quad$ il V; a Spoleto e Nami il IV.

* 20 giugro 1891 - Cittaducale (scossa di IV-T).

$I_{0}=I V-V$

$1 \cong 6 \mathrm{~km}$

* 22 gennaio 1892 - Colli Laziali - Questo terremoto (di griulo VIII) $I_{0}=$ VIII con epicentro tra Genzano e Telletri ebbe una vastia $1 \cong 78 \mathrm{~km}$ estensione macrosismica. Pel quanto la sua di$I_{P}=I V \quad$ stanza dalle Sorgenti del Peschiera sia tra le maggriori da noi prese in considerazione, non possiamo a meno di notare che l'intero percor'so dell'Acquedotto del Peschier"a (per altro non ancora costruito a quell'epoca) si trova in piena aerea macrosismica (v. fig. 6) a che la stessa zona di Cittaducale, Sorgenti e.c.... fu interessata per un IY-III della Scala Mercalli-sieberg.

***: 2 agosto 18.9.3 (ore 02 (a.) - Montereale - Scossa di VI a Montereale. $I_{1 "}=$ VI La propagazione è piuttosto anomala, come ben si $A \simeq 2.5 \mathrm{~km} \quad$ rede dalla fig. 7 , tuttavia ad Antrodoco fu sentita $I_{P}=$ Ir di $\mathrm{Y}$, ed a Cittaducale, Rieti, Fonti del Peschiera, di $I Y$. 
Tarie scosse prima e poi, ma tutte esamitesi, pare, tra il giomo 1 e il giorno 2 agosto.

****: 28 giugno $7898\left(00^{\mathrm{n}} 38^{\mathrm{m}}\right)$ - Ricti - Preceduta da varie scosse $I_{0}=$ IX $\quad$ che iniziarono fin dal febbraio 1898 (di IV; IV-III; $\Delta \cong 14 \mathrm{~km} \quad$ III; II-III), la scossa del 28 giugno fu di vasta por$I_{P}=$ V-VI tata. Allepicentro, localizzato tra Rieti e CoppaelliBasso (località quest'ultima interamente distrutta) l'intensità può valutarsi di IX. A Cittaducale di VIII, alle Fonti di V. L'andamento delle isosiste (v. fig. 8) ricorda molto da vicino quello del terremoto oggetto del nostro studio, con un accentuato spostamento dei gradi più bassi in direzione sud-ovest fino ard includere Roma, Frascuti, Velletri nella isosista di IV.

A questa scossa fecero seguito numerose repliche di minore intensità, che perdurarono anche nei mesi di luglio, agosto, settembre.

Si può riassumere l'andamento di questo periodo sismico nel quadro seguente:

Scosse premonitorie:

8 febbraio 1898 - alle $3^{\text {n }} 57^{m}$ una scossa di IV presso

Rieti (sentita anche a Cittaducale)

8 febbraio - alle $04^{\text {n }} 10^{\mathrm{m}}$ una scossa di III presso Rieti

8 febbraio - alle $04^{\mathrm{n}} 20 \mathrm{~m}$ una scossa di III presso Rieti

ai primi di marzo qualche scossa di $\amalg$ presso Rieti

$2 \tau$ giugno - alle 01" ma scossa di III presso Ricti

28 giugno - alle $00^{\mathrm{n}} 30^{\mathrm{m}}$ una scossa di IV pressso Rieti

Scossa principale:

28 giugno $1898-00^{\mathrm{n}} 38^{\mathrm{m}}-$ scossa di $\mathrm{IX}$

Repliche inportanti:

28 giugno - $01^{\mathrm{h}}$ a. - scossa di III a Rieti

29) giugno - $04^{14} 56^{\mathrm{m}}$ - scossa di IV a Rieti,

di III a Citfaducale

.. nel mese di luglio altre scosse ...

30 agosto $-17^{\mathrm{h}} 30^{\mathrm{m}}$ cal scossa di VI a Cittaducale

9) settembre - 20 13m ca. scossa di VI a Rieti, di V-IV a Cittaducale. 
Ii interessante ricordare quanto il Baratta rlice a proposito del "Distretto sismico del Felino" (pag. i62 a seguenti del "I terremoti in Italia "), riassumendo a grandi linee la situazione sismica della zona: "In quest" zona dobbiamo distinguere tre centri principali: uno si"tuato alla confluenza del Velino con la Nera, ossia l'area dei terremoti "di Piedilueo; gli altri due sono posti rispettivamente nei pressi di Rieti " $e$ di Cittaluscale.

"I terremoti del 178j mostrarono - almeno le manifestazioni may"giori - il loro centro a Piediluco, che insieme a Papigno ed a Buon"acquisto rimase in gran parte distrulto...

"La zona sismica di Rieti fu colpita da manifestazioni corocentriche "nel 29 maro 1rif ed al 2:2 maro 18:21. Questa città poi nel 1898 fu con"quassata da un violentissimo terremoto, il cui centro pare sia stato nei "pressi di Goppalli, che si illentifica con $i$ due precedentemente ricordati . .

"Lne delle repliahe del parossismo del $1 \% 03$ (quella del 1:5 febbraio "ore or) fu corocentrica a Rieti, localitì dhe pin dogmi altra ebbe a soffrirme "tristi conseguenze...

"Negli ultimi terremoti reatini del 1898 Cittaducale risenti minori "rocine che non Rieti: nel 1703 ebbe qualche cusa caluta e molte lesionate "e rese inabitabili mentre le vicine localita di Pendenza (a km 7 ) e di An"broloco (a lim 27) rimasero distrutte. Ė probabile che abbia sofjerto anche "in occasione dei terremoti del $1+61$...

"Cittalucale nel l:50:2 fu traralluta per to giomi da rovinosi scuo"timenti, o da altri fortissimi nel 1:58:. Questi ci riveluno la presenzu di "uno speciale centro, le cui manifestazioni non si possono identificare con "quelle del vicino focolare reatino. Oltre alla magyiore manifestazione si"smica del 1898 . . muove prove ci porgono aleune repliche susseguenti: "quella del 30 agosto (ore 18 circa) fu molto pin forte a Cittuducale che non " a Rieti: un'altra invece, avvenuta nella sera del I sett. riuse" pin intensa "nell'ultima locelitì che non nella prima. Il centro di Cittaducale. La dato "luogo " parechie manifestazioni lievi-forti tra le quali. . 1842 now. 2:5; "18.5.) sett. 24; 18.56 marzo 10: 1881 ayosto 14 e nov. 18; 1891 giugno 20, "sec....".

Secondo l'autore sicehe nel distretto del Velino si avrebbero tre centri sismici (Piedilueo, Rieti, Cittalucale) distinti.

Questo potrebbe anche assere vero, però, a nostro avviso, essi non sarebbero mai inclipendenti, giacché la loro vicinanza stessa fa presumere un processo di solleceitazioni a catena che renderebbe difficile la esatta discriminazione dei fenomeni. 
Continuando la ricerea storica delle manifestazioni telluriche troviamo che al periodo sismico precedentemente segnalato se ne sorrappone un altro, di altra provenienza, ma che egualmente interessa in maniela piuttosto vivace la zona del Reatino. Ed infatti il

***:* 25 agosto 1898 - alle $17^{\mathrm{h}} 37^{\mathrm{m}}$ ea. con epicentro nei dintorni di $I_{0}=$ VII Visso si ebbe una scossa abbastanza forte per la qua$\Delta \cong 60 \mathrm{~km}$ le si hamo le seguenti notizie macrosismiche:

Sentita di VII a Rasemna, Mevale, Chiusita (Visso), Renalo (Sellano);

di VI a Precei;

di $V$ a Visso, Sellano, Cerreto, Foligno;

di IV a Rieti, Spoleto, Samano, Caldarola. Cerreto;

di III a Bolognola, S. Severino, Macerata, Cupramontana, Leonessa, Cantalice, Magliano Sabino.

Numerose repliche (di III-IV) si ebbero per circa un mese, tra cui, particolarmente menzionate quelle del:

9 sett. (23" $30^{\mathrm{m}}$ ('at) $)$

10 sett. $\left(14^{\prime \prime}+5^{m}\right)$ e la più forte di tutte quella del

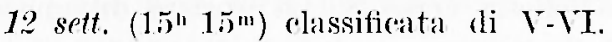

***** Il 19 muygio 1900 alle ore 18, ron epicentlo ad Arrone (Terni) $I_{0}=\mathrm{VI} \quad$ una scossa di VI fa seguito ad un periodo sismico $\Delta \cong 30-35 \mathrm{~km}$ che perdurava già da alcuni mesi. Ad essa seguono numerose repliche, tra cui le principali sono:

20 maggio $1900\left(8^{11}+5^{\mathrm{m}}\right): \mathrm{IV}-\mathrm{V}$ ad Arrone,

27 maggio (03 $\left.08^{\mathrm{m}}\right): \mathrm{V}$ ad Alrone, 28 maggio $\left(04^{h} 05^{m}\right)$ : III-IV a Spoleto, 28 maggio $\left(09^{\mathrm{n}} 14^{\mathrm{m}}\right)$ : III-IV a Spoleto, 28 maggio (23" (а.) : III-IV a Spoleto, 29 maggio $\left(00^{\prime \prime} 03^{\mathrm{m}}\right)$ : III-IV a Spoleto 29 maggio (0.th $\left.07^{m}\right)$ : III-IV a spoleto, 30 maggio $(22120 \mathrm{~m})$ : III-IV ad Arrone, 1 giugno (20" 30m): IV a Spoleto, 3 ginguo $\left(15^{1-} 52^{\mathrm{m}}\right)$ : III a spoleto,

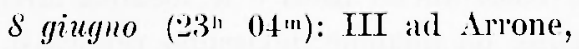
15 giugno (05" 51m): III ad Arrone. 
Questa la parte più appariscente del periorlo che interessò la zona: sull'attività che precerdette la scossa piì forte, (quella del 19 magrgio) mancano purtroppo notizie particolaregrgiate: commque si parla di ... mesi di prejarazione.

*** 23 ottobre $1902\left(09^{n} 51^{m}\right)-$ VI-VII all'epicentro: Rieti.

$I_{o}=$ VII-VI Anche questa è la scossa più importante di mo pe$\Lambda \cong 10 \mathrm{~km}$ riorlo sismico piuttosto breve che però si presentò a $I_{P}=\mathrm{V} \quad$ cavallo di movimenti precerlenti e susseguenti piuttosto sensibili. Infatti la scossal che la preceedette, quella del

21 ottobre alle $19^{\mathrm{h}} 04^{\mathrm{m}}$ fn probabilmente di VI all'ejirentro e fu sentita lievemente perfino a Roma e Roeca di Papa. Poi vemme la principale, quella del

2:3 ottobre $\left(09^{\mathrm{n}} 5 \mathrm{1}^{\mathrm{m}}\right)$ che provocò crolli e lesioni a Rieti e Cittaducale. Fu averetita entro una vasta area comprenclente anche parte relle provincie di Roma, L'Aquila, Terni, Perugia, fino ard Assisi verso Norrl, e Velletri rerso $\mathrm{S}$. Iue isosiste disegnate nella fig. 9 si limitano a quella di $V^{\top}$ perché mancano notizie dettagliate per poter disegnare quelle di grato meno intenso, tuttavia de ben visibile come la zona di intensità più elevata sia molto prossima alle Sororenti del Peschier"il.

A questa seguirono varie repliche la più importante delle quali fu quella del:

2.5 ottobie alle $9^{\mathrm{n}} 35^{\mathrm{m}}$, relassificata con un $Y^{\circ}$ all'eppicentio.

**** 22 dic. 19910 (13" 34m (ea.) (on epicentro nei pressi di Accumoli: $I_{0}=$ VII-VI $1 \mathrm{Im} \mathrm{VI+}$

$\Delta \cong 10 \mathrm{~km} \quad\left(18^{\mathrm{h}} 18 \mathrm{~m}\right.$ (a.) con epicentro nei pressi di Accumoli: un VI (al. a poi il

26 diember 1910 (17" $30 \mathrm{~m}$ (a.) con epoicentro nei pressi di Acenmoli un VI-VIL

Queste tre forti scosse, che interessarono per i grarli più bassi anche Rieti a le località circostanti, furono seguite da qualche lievissima replica il giomo 27 die. (ore 0:5 (a.) semalata prevalentemente a Montercale. 
*:*: 13 grnmaio $1915\left(7^{\mathrm{h}} 52^{\mathrm{m}}\right)$ Epicentro nella Marsica (XI-XII all'e$I_{0}=$ XI-XII picentro).

$4 \cong 50 \mathrm{~km} \quad$ Questo immane ataclisma (he molti italiani ancora $I_{P}=$ VIII ricordano, pur non arendo avuto la sua origine nelle immediate vicinanze della zona del Peschiera, ha tuttavia interessato la zona stessa per un VIII-IX grado della Scala Mercalli-Sieberg. Il linguaggio delle isosiste nella fig. $10\left(^{1}\right)$ è estremamente chiaro. La città di Avezzano fu completamente distrutia, ma si puó dire che quasi l"intero territorio della penisola italiana sia stato sollecitato macrosismicamente, e di fronte ad episodi cosi sconvolgenti a di cosi rasta portata non ha più senso, ovviamente, parlare di sismicitì locale. Tuttavia anche la conca dol Fucino, sia pure, fortunatamente, in proporzioni molto più modeste di questo terremoto del 1915, è sede di manifestazioni sismiche che talvolta possono turbare, marginalmente, la zona del Peschiera a del Reatino.

E naturale che ad un fenomeno cosi imponente (ma che peró sorse improrviso a quanto pare senza preannunci di sorta) sia seguito un lunghissimo periodo di assestamento. L'equilibrio, cosi profondamente turbato, ha ripreso la sua relativa stabilità dopo innumerevoli repliche a dopo molto tempo (almeno $t$ anni). Soltanto nelle prime $2 t$ ore che seguirono la catastrofe furono registrate 290 repliche, e 1280 furono segnalate nei primi 6 mesi: molte di esse furono arvertite dalle persone e parecehie, fino alla fine del mese di gemnaio 1915, provocarono anche nuovi damni.

* Il 15 febbr. 1915 $\left(06^{\mathrm{n}}+\mathrm{i}_{\mathrm{m}}\right)$ si ha notizia di un terremoto con epi$I_{0}=\mathrm{VI}$ centro a Cittaducale, di intensità $I_{0}=$ VI. Mancano $\Delta \cong 6 \mathrm{~km}$ però notizie dettagliate.

**** Lill $1100.1915\left(04^{11}-10^{\mathrm{m}}\right)$ iniziò un periodo sismico che si esauri $I_{0}=$ VI-VII in un tempo molto breve (appena dne giorni) però $A \cong 30-35 \mathrm{~km}$ con una noterole frequenza di piccole repliche.

(1) Le isosiste di questo teremoto ai sono state gentilmente fornite dal prof. Mario De Pantilis. 
Ta scossa principale (quella dalle $04^{\mathrm{h}} 40^{\mathrm{m}}$ ) ebbe per epientento:

Sitroncone: ivi la intensità fu di VI-VII:

a Terni di VI:

a Ricti, Cottanello, Montasola di V;

ad Attigliano, Magliano Sabino, Orte, Sangemini, Arrone, Narni, Papigno di IV;

ad Amelia, Baschi, Cammara, Poggio Mirteto, Soriano al Cimino di III.

Una trentina di repliche tra il II ed il IV grado Meralli si rbbero nei giorni 11 e 12 novembre.

***** 22 aprile $1916\left(05^{\mathrm{n}} 33^{\mathrm{m}}\right)$. Epicentro I'Aquila-Tormimparte $I_{0}=\mathrm{VII} \quad I_{0}=\mathrm{VII}$.

$\Delta \cong 30 \mathrm{~km} \quad$ Ci troviamo ancora di fronte ad un periodo sismico $I_{P}-$ IV-III notevole più per la frequenza delle scosse che per la intensità: questo terremoto à la massima espressione di un'attività iniziata fin dal febbraio. Feco il quadro riassuntico:

Scosse precedenti:

1.3 febbraio 1916 - Varie scosse tra il III ed il V grado Merealli-Sieberg;

14 marzo $\left(01^{\mathrm{h}} 39^{\mathrm{m}}\right)-I_{o}=$ VI. Tomimparte (VI), L'Aquila, Carsoli, Gubbio, Subiaco (di V-IV);

21 aprile $\left(13^{\mathrm{n}} 46^{\mathrm{m}}\right)-I_{o}=$ VI. L'Aquila, Tornimparte (di VI) Scoppito (di IV) Popoli, Todi, Roccar di Cambio (di III);

Scossa principale:

22 aprile $\left(05^{\mathrm{h}} 33^{\mathrm{m}}\right)-I_{o}=$ VII. (L'Aquila, Tornimparte). Per questo sisma (v. fig. 11) le Fonti clel Peschiera furono interessate marginalmente dalla isosista di IV. Scosse successive:

Numerosissime repliche (di III-IV) si ebbero per parechi mesi. Esse cessarono solo verso la fine del novembre 1916.

*** 4 luglio $1916\left(06^{\mathrm{n}} 06^{\mathrm{n}}\right.$ (a.) $-I_{o}=$ VII-VII (Arquata del Tronto). $I_{o}=$ VII-VIII Preceduta di un'ora da una scossa piuttosto forto $\Delta \cong 48-50 \mathrm{~km}+$ luglio $-05^{\mathrm{h}} 21^{\mathrm{m}}\left[I_{o}=\mathrm{VI}-\mathrm{VII}\right]$, quella del 
$I_{p}=\mathrm{IV} \quad$ \& luglio $-06^{\mathrm{n}} 06^{\mathrm{m}}\left[I_{0}=\right.$ VII-VIII $]$, fu la pì̀ forte. Ad Arquata del Tronto, zona epicentrale, l'intensità macesismicar raggiunse il VII-YIII grado. Te isosiste della fig. 12 mosti"ano chiammente come le Fonti del Peschiera furono ampiamente contenute entro la curva di IV.

Numerose repliche si ebbero durante la giomata: le più forti sono le tre seguenti:

t luglio $-17^{\mathrm{h}} 05^{\mathrm{m}}$ di V-VI all'epicentro (Ussita a provincie dell'Aquilat e Perugial);

t luglio $-23^{\mathrm{n}} 01^{\mathrm{m}}$ di VI allepicentro (Al'quata del Tronto e provincie di Ancona, L'Aquila, Ascoli P., Macerata, Perugia).

4 luglio - $23^{\mathrm{n}} 07^{\mathrm{m}}$ - di VI all'epicentro (Arquata del Tronto e provincie di Ancona, L'Aquila, Ascoli P., Macerata, Perugia).

Anche questo fu un periodo breve ma pintitosto intenso.

**** 16 nov. $1916\left(07^{\mathrm{H}} 35^{\mathrm{m}}\right)-I_{o}=$ VII-VIII (Cittameale, Rieti, Tro$I_{0}=$ VII-VIII gnano).

$\Delta \cong 30 \mathrm{~km} \quad$ Inche per questo terremoto le Sorgenti del Peschiera. $I_{P}=I V-V$ si trovarono interessate da un IV-V grado (v. fig. 13). Esso fu seguito da qualehe replica di IV, e poi da ma piì forte il

22 nov. $1916\left(11^{\mathrm{l}} 20^{\mathrm{m}}\right)$ sentita di $\mathrm{V}$ all'epicentro o di IV-III ad Accumoli, Amatrice, Visso, Giano, Poggiodomo.

Anche questo periorlo si esaurì nel giro di pochi giorni.

* Il 13 maggio $1923\left(1^{\mathrm{n}} 31^{\mathrm{m}}\right)$ si ebbe una scossa isolatia (o per lo $I_{o}-$ VI meno non segnita da repliche sensibili) nell'Aquilano. $\Delta \cong 30 \mathrm{~km} \quad$ Presso Borgo Collefegato, alle falde del Velino, si $I_{P}=$ IV ebbe il massimo dell'intensità (VI); nessm altro paese subi damni, però il movimento ebbe una vasta estensione di sensibilità all'uomo: (investì di IV le Fonti del Peschicera, a giunse di III fin oltre Roma ( $r$. fig. 1t). 


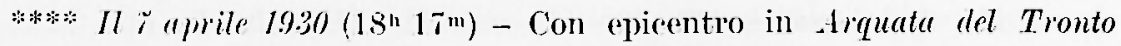
$I_{0}=$ VI avvenme un terremoto di VI, che si propagò di V-VI

$I \simeq 45-50 \mathrm{~km}$ ad Accumoli, Norcia, Cascia, ed interesso di IV anche le province di Rieti, Ascoli Piceno, Nacerata, Perugia, Terni, Teramo.

Ancora trattasi di un periodo sismico che si esaluri con varie repliche di III-IV per una quindicina di giorni.

* Il 12 agosto $19.98\left(033^{\mathrm{n}} 30^{\mathrm{m}}\right)$ I'Aquila fu colpita da una scossa di VIo. $I_{0}=\mathrm{VI}$ Larea macrosismica, piuttosto estesa (v. fig. 15) in$1 \cong 30 \mathrm{~km} \quad$ clude le Fonti del Peschiera nella isosista di V-VI. $I_{P}=\mathrm{V}-\mathrm{VI}$

* Il 16 jebl, $1940\left(03^{1}\right.$ 05m) una scossa di IV-V colpì Ricti ed Antro$I_{0}=\mathrm{IV} \cdot \mathrm{V}$ doco. Mancano notizie particolareggiate.

$1 \cong 5 \mathrm{~km}$ ?

Da questo momento e per diversi anni le notizie che si sono potute racogliere sono frammentarie ed incomplete. Gli eventi bellici hamno influito notevolmente sulla regolarità e l'efficienza del servizio macrosisnico a microsismico per cui si può senz'altro ritenere che il decennio 1940-1950 così come a qui esposto non rispecehi completamente l'eflettiva siturzione sismica.

* Il 20 giugno $1942\left(07^{11} 48^{m}\right)$ un IV fu segnalato a Lidquila, Monte$I_{0}=$ IV reale, Sassa.

$1 \cong 25 \mathrm{~km}$

*Il + nor. $194:\left(03^{\mathrm{n}} 19 \mathrm{~m}\right)$ un IV-V a $L$ I Aquilu, Sassa.

$I_{0}=\mathrm{IV}-\mathrm{V}$

$1 \cong 2.5 \mathrm{~km}$

** Il 29) gemm. 194.3 (04 $\left.24^{\mathrm{m}}\right)$ un VI interessò Arquata del Tronto, $I_{0}=\mathrm{VI} \quad$ Areumoli, Cittareale.

$1 \cong 40-45 \mathrm{~km}$ Nella fig. 16 si verle come Cittalucale, Rieti, le Fon$I_{l}=$ IV ti del Peschiera siano racchinse entro la isosista di IV. Seguirono alcume repliche: una di IV alle $04^{\text {" }} 36^{\text {ra }}$ ed una di III alle $08^{\mathrm{h}} 58 \mathrm{~m}$ del giorno successivo. 
* + gennaio $1949\left(20^{\mathrm{n}} 55^{\mathrm{m}}\right)$ Si ha notizia di un VII-VI sentito a Ri$I_{0}=$ VII-VI codutri (Rieti), ma mancano ulteriori informazioni. $1 \cong 20 \mathrm{~km}$

* 199 giugno $194.9\left(15^{\mathrm{n}} 33^{\mathrm{m}}\right)$ a Ricodutri (Rieti) sentito di IV.

$I_{0}=\mathrm{IV}$

$1 \simeq 20 \mathrm{~km}$

* Il 2r oftobre 194.9 (21." $\left.08^{\mathrm{m}}\right)$ una scossa piuttosto importante fu I. … V-YI? sentita a Temi, Rieti e province, ma non si lanno notizie particolaregggiate.

$* I l \div$ ma:o $19: 50(0.4 \mathrm{n} 49 \mathrm{~m})$ Cittaducale, Casette, furono interessate da $I_{0}=$ IV una scosssa di IV, eui segui una replical alle $05^{1 \mathrm{n}} 25^{\mathrm{m}}$ $A \simeq 5-7 \mathrm{~km} \quad$ dello stesso eriomo.

* 12 maro 19:50 (19" 15 $\left.15^{\mathrm{m}}\right)$. Ad Accumoli (Rieti) $I_{o}=$ VII ca. (man$I_{0}-$ VII ano altre notizie).

$1 \simeq 40 \mathrm{~km}$

*:*: j) sett. 19:50 $\left(05^{\mathrm{h}} 10^{\mathrm{m}}\right)$ con epicentro nel Ciran sasso un forte ter$I_{0}=$ VIII remoto di $I_{0}=$ VIII ebbe vasta propagazione. Tale $A \simeq 28 \mathrm{~km} \quad$ terremoto fu fatto oggetto di studio particolareggiato $I_{P}=$ VII $\quad$ a la rete delle isosiste che ne fu traceiata e che qui liproduciamo nella tig. 17 mette in evidenza come per questo sisma le Fonti del Peschiena siano state raggiunte da un VII Mercalli-Sieberg e come inoltre lintero pereorso dell' Arequedotto si sia trovato in pienal area macrosismica.

Questo terremoto is stato preceduto da luna forte scossa e seguito, nel griro di poche ore, da numerose repliche di intensità molto minore.

** $/ 118$ sett. $19.50\left(01^{\mathrm{h}}+\left(0^{\mathrm{m}}\right)\right.$ si ha notizia di un VIo sentito a Monte$I_{o}=:=$ VI reale e di una replica alle $01^{\text {h }} 59^{\mathrm{m}}$ dello stesso giomo. $1 \simeq 26-28 \mathrm{~km}$

* 28 maggio 19:51 $\left(08^{11} 36^{\mathrm{m}}\right)$, a C'ittaducale, Casette, Cottanello is stata $I_{0}=$ IV sentita una scossa di IV e di III a Rieti.

$1 \simeq 10 \mathrm{~km} ?$ 
*** L'8 agosto 19.jl, alle 211" 56m, ron epirentro in provincia di Te$I_{o}=$ VUI-VII ramo ebbe lugo una forte scossa di terremoto. La $A \cong 68 \mathrm{~km}$ intensità massina $I_{0}$ si ebbe a Canzano (VII-VIII). $I_{p}=$ IV I'epicentro di questo sisma è, tra quelli da noi presi in esame, uno dei più lontani dalle Sorgenti del Peschiera (vedi fig. 18), tuttavia le Sorgenti stesse sono compresesentro la isosista del IV grado. Il movimento fu distintamente arrertito di III anche a Subiaco, llatri ed Assisi.

A questo seguirono numerose replicke nei griorni $8-9$ agosto: quelle registrate dall'osservatorio di Roma sono le seguenti:

$\begin{array}{ccccc}8 \text { aggosto I) all. } & 22 " & 11 \mathrm{~m} \\ \text { II) } & " & 23 & 09 \\ \text { III) } & " & 23 & 53 \\ 9 \text { agosto } & \text { IV) } & " & 00 & 59 \\ \text { V) } & " & 05 & 45 \\ \text { VI) } & " & 06 & 45 \\ \text { VII) } & " & 19 & 13\end{array}$

* Il 26 febbr. 19.5.3 $\left(08^{\mathrm{h}} 34^{\mathrm{m}}\right)$ con epicentro tra Honteleone di spoleto, $I_{0}=\mathrm{V} \quad$ Ferentillo, Leonessa fu avvertita una scossa di V. $\Delta \simeq 28 \mathrm{~km} \quad$ Mancano però altre notizie.

** Il 16 agosto 19:56 $\left(09^{\mathrm{h}} 3 \tilde{5}^{\mathrm{m}}\right)$ con $I_{0}=\mathrm{V}$ fu arvertita una scossal $I_{0}=\mathrm{V} \quad$ a Cittareale, Santa Giusta, Santa Croce, Trimezzo. $\Delta \simeq 28 \mathrm{~km} \quad$ Essa fu strettamente locale, con limitata estensione macrosismica. Una replica di IV-Y la segui poche ore dopo (12/ 23m).

* 25 gennaio $195 \%$ (05 $\left.40^{\mathrm{m}}\right)$. Epjocentro tra Pizzoli e Barete, in pro$I_{0}=\mathrm{VI}-\mathrm{Y}$ vincia dell'Aquila. Landamento delle notizie macro$\Delta \cong 24-25 \mathrm{~km}$ sismiche $\dot{a}$ il seguente:

VI-Y a Pizzoli e a Barete;

Ir a Montereale, Capitignano, Cagnano Aniterno, Tornimparte, Rocea di Mezzo, Caporciano, Ofena, L'Aquila (e frazioni), Isola del Gran Sasso (e commini), Tossicia, Montorio al Vomano, S. Cosma, Cesarproba; 
III a Cittareale, Borbona, Rocea di Corno, (prov. Rieti), Ovindoli, Rovere, Capestrano:

III-II a Posta, Antrodoco, Amatrice.

* 12 mara 19:3\% (17" 20m) Epicentro presso Norria.

$I_{0}=\mathrm{VI} \quad$ Isosiste:

$d \cong 45 \mathrm{~km} \quad$ VI a Norcia e circondario;

Y a Cascia, Arendita, Piandoli, Roceaporena, Monteleone di Spoleto, Trivio, Ruscio, Castel S. Giovanni, Ualtignano, Onelli;

IV a Preci, Paterno, Abeto, Collazzoni, Polino, Arrone, Colle S. Angelo, Morro Reatino, Leonessa, Albaneto, S. Giusta, Cittareale, Trinezzo;

III a Pierdiluco, Laabro, Rivodutri, Cantalices, Sigillo. Posta, Montereale, Mmatrice.

* 73 meroo $79.5 \%$ (04" 19m): una scossa di $\mathrm{V}$ all'epicentro.

$I_{o}-\mathrm{V} \quad \mathrm{V}$ a Cascia, Monteleone di Spoleto, Amatrice;

$A \cong 35 \mathrm{~km} \quad$ IV a Preei, Leonessar;

III a Vallo di Nera, Arrone, Polino, Montefinanco, Cittareale, Posta, Grececio, Montereale...

*: L:11 "prile 195\% (17" 19m) un tepremoto con epicentro tra Pe$I_{0}=$ VI trella salto a Fureo sabimo, interesso sensibilmente la $\Delta \cong 10 \mathrm{~km} \quad$ zona del Peschiera. Il VI delleppicentro si smorzò $I_{V^{2}}=-\mathrm{V} \quad$ in $\mathrm{m} \mathrm{V}$ alle Fonti del Peschiera e ad Antrodoco per giungere eon un IV a Cittaducale. La carta della fig. 19 rappresenta chiaramente l'estensione " l'andamento delle isosiste. Ancoma una volta ci troviamo in presenza di una aceentuata deformazione dei gradi bassi, in direzione di Roma.

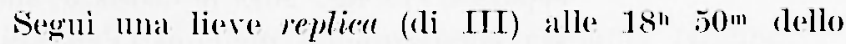
stesso giomo.

* 8 frbbraio 19:58 $\left(07^{\prime \prime} 00 \mathrm{~m}\right)$ : una scossa di:

$I_{o}=$ VI-V VI-Y tra Stroncone e Greccio, sentita di

$A \simeq 27 \mathrm{~km} \quad V$ a Terni, di

IV a Mamore, Piediluco, Collescipoli. 
* 8 muggio 19:8 (01" $\left.13^{\mathrm{m}}\right)$. I a zona epicentmale è Monteleone di Spo$I_{1 "}=\mathrm{V}$-VI leto, dove la scossa è stata sentita di V-VI Mercalli$\Delta \cong 30-35 \mathrm{~km}$ Sieberg. È stata altresi sentita di $V$ a Cascia, di IV a Norcia e Vallo di Nera, di III a Preci.

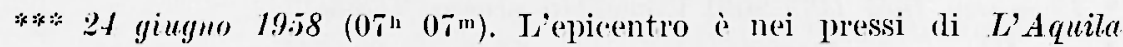
$I_{0}=$ VII $\quad$ dove il terremoto è stato sentito di VII: anche questa $A_{p} \simeq 30 \mathrm{~km} \quad$ volta le manifestazioni macrosismiche giunsero fino a Roma dove fu averertito un IV-III grado.

Nella stessa giomata del 24 giugno altre scosse di eguale provenienza e di minore intensità furono segmalate, prima e dopo quella delle $07^{\mathrm{n}} 07^{\mathrm{m}}$ :

I) premonitoria alle $05^{\text {h }} 24^{m}$

II) " " $\quad 0528$

I) replicar alle $09^{\mathrm{n}} 08^{\mathrm{n}}$

II) " $\quad 14 \quad 25$

III) " " $\quad \mathbf{1 5} \quad 51$

IV) $\quad$ " $16 \quad 29$

* 72 marao $1960\left(01^{\mathrm{h}} 31^{\mathrm{m}}\right)$. Tiepiecentro d la zona di cittarcale dove $I_{0}-\mathrm{V}$ la scossa $\dot{e}$ stata sentita di V. A Norcia, Lconéssa, $1 \simeq 28 \mathrm{~km}$ Amatrice di IV; a Posta, Monteleone di Spoleto, Cascia, Vallo di Nera di III.

Data la relativa esiguita del fenomeno mancano notizie più numerose, le quali invece sono abbondanti per il terremoto seguente arvenuto press'a poco nella stessa zona, ma più forte.

* 16 maro $1960\left(02^{\prime \prime} 53^{m}\right)$. I paesi in cui la scossa è stata sentita più $I_{0}=\mathrm{VI}$ intensamente sono Cittareale e Montemonaco (di VI) $I_{I^{\prime}}=$ IV tra i quali però cadono delle località in cui la percezione è stata di $\mathrm{V}$, talché i due sesti gradi rinangono separati (v. fig. 20): le isosiste successive poi però si regolarizzano, e quella di IT go grado include le Fonti del Peschiera, Antrodoco e Cittaducale.

***:* Il 2.3 aprile 1960, alle $12^{\mathrm{h}} 45^{\mathrm{m}}$, con un terremoto sentito di VI $I_{0}=\mathrm{VI}$ ad Acquasparta si hanno i prodromi di quel periodo $d \simeq 50-55 \mathrm{~km}$ sismico ehe successivamente, dal mese di luglio interessò a lungo Terni e provincia. A seguito delle scosse 
più forti l'Istituto Sazionale di Geofisica provide ad installare una stazione sismica provvisoria d'osservazione che funzionò per mesi (prima a San Gemini. poi a Terni) sotto il diretto controllo dei tecnici della sede di Roma.

Il funzionamento di detta stazione, protrattosi fin oltre il dicembre dello stesso anno, consenti la raccolta di una completa documentazione locale interessante a fini di uno studio esauriente sulla sismicita di Terni e circondario (studio in programma dagli autori del presente lavoro); tuttavia, per quanto gli epicentri di questi sismi siano abbastanza vicini alle Fonti del Peschiera, pure queste non sono mai state raggiunte dagli effetti macrosismici, neanche delle scosse più forti; talché non riteniamo opportuno entrare in maggiori dettagli.

* Il \pm dicembre $1960\left(05^{\mathrm{h}} 30^{\mathrm{m}}\right)$ una scossa che ebbe il suo epicentro $I_{o}=$ V-VI poco a Nord Est di Spoleto, con una $I_{0}$ di V-YI, si $1 \cong 50 \mathrm{~km}$ propagò fin oltre le Fonti interessando queste per $I_{P}=$ IV un IV Mercalli-Sieberg (v. fig. 21).

* 2.5 mar:o 1961 ( $\left.11^{\mathrm{h}} 40^{\mathrm{m}}\right)$ in tutto il territorio di Pizzoli (L'Aquila) $I_{o}=\mathrm{V} \quad \mathrm{fu}$ arvertita una scossa di $\mathrm{V}$ grado Nercalli-Sieberg. $1 \cong 25 \mathrm{~km}$

*: 12 aprile 1961 $\left(01^{\mathrm{h}} 43^{\mathrm{m}}\right)$ una scossa piuttosto forte fu segnalata di $I_{0}=$ VI VI a Roca di Botte e Peretto (L'Aquila).

$1 \simeq 35 \mathrm{~km} \quad \mathrm{Fu}$ inoltre sentita di: $V$ ad Oricola (L'Aquila) e Vicovaro (Roma):

IV a Scanzano, S. Stefano, S. Giovanni, Castelvecehio, Carsoli, Cappadocia (L Aquila), Arsoli, Riofreddo, Saracinesco (Roma), Turania (Rieti);

III a Tiroli, Marcellina, Castel Madama (Roma). Questa scossa ela stata preceduta da una di intensità quasi uguale, il 10 aprile $\left(06^{\mathrm{h}} 55^{\mathrm{m}}\right)$, e fu seguita da un paio di repliche meno intense.

Fino al gingno dello stesso anmo non si hamno altre notizie: se attività c'è stata essa deve aver raggiunto proporzioni molto modeste, sì 
da sfuggire all'attenzione macrosismicar ed al controllo microsismico dell'Osservatorio di Roma. Invece dal giugno 1961 in poi la docmmentazione di qualsiasi attività della zona del Peschiera si fa molto più minnziosa, poichó in tal data, come si è detto, fu installata una terna di sismografi opportumamente costruiti per la registrazione dei terremoti di carattere locale. Ia stazione, che funziona giornalmente ormai da più di un anno e mezzo ha consentito di esaminare, diremo cosi, al microscopio la sismicita locale. Già nella prima parte del nostro lavoro abbiamo pubblicato la documentazione del semestre giugno-dicembre 1961: nella prossima (che seguirà la presente), completeremo il biennio giugno 1961 -giugno 1963. Nessumo studio, per aceurato (he sia, potrà fornire elementi più completi a più esaurienti di questi.

Intanto, però, scorrendo tutta la documentazione che abbiamo esposto nel presente lavoro, possiamo dire di avere ma visione abbastanza completa della sismicità della regrione.

I PERIOI)I SISHICI.

Abbiamo già messo in rilievo come ma percentuale piuttosto alevata dei fenomeni tellurici che nel corso dei secoli lanno interessato la regione in esame si sia presentata con le calatteristiche di periodi sismici.

Il fatto ci à semblato degno di rilievo, ed abbiamo voluto pertanto porre una particolare attenzione su quelli, tra i tanti periodi incontrati, per i quali ì stato possibile avere notizie abbastanza dettagliate ed esaurienti. E superfluo far notare (he anche di questi tuttavia le notizie sono incomplete, sfuggendo alla cromaca i particolari di minor rilievo.

I periodi per i quali (i siamo particolarmente interessati sono, nellordine cronologico, i seguenti:

I) novembre 1461-febbraio 1462: zona epicentmale L'Aquila;

II) ottobre 1599-gennaio 1600: zona epicentrale Cascia-Norcia;

III) 8-17 ottobre 1639: zona epicentrale Amatrice;

IV) ottobre 1702-1703-febbraio 1704: zona epicentrale CasciaNoreia-I'Aquila;

V) ottobre 1785-agosto 1786: zona epicentrale Piediluco;

VI) settembre-ottobre 1878: zona epicentmale Fonti del Clitunno ([imbria); 
VII) marzo 1881: zona epicentrale Umbria;

VIII) febbraio-settembre 1898: zona epicentrale Rieti;

IX) febbraio-giugno 1900: zona epicentrale Arrone;

$\mathrm{X})$ febbraio-dicembre 1916: zona epicentrale L'Aquila-Tornimparte.

Per la parte descrittiva di questi periodi rimandiamo a quanto precedentemente esposto. Le figure da 22 a 31 esprimono graficamente lo svolgimento di ciaseuno di questi eventi (in ascisse sono riportate le date e in ordinate le intensità in gradi Mercalli delle singole scosse). Valendosi poi della relazione che lega la massima intensità epicentrale $I_{o}$ di una scossa, all'energia sismica erogata dalla scossa stessa abbiamo calcolato, per ogni periodo, l'energia sviluppata volta per' volta dalle singole scosse (o, specie per le più piccole, di un certo numero di esse, numero valutato grossolanamente a seconda delle notizie cumulative raccolte). Siamo stati così in grado di calcolare l'energia totale sviluppata durante ogni intero periodo, e per ciascun periodo anche l'andamento di questa erogazione. I grafici delle figure 22a-31a su scala logaritmica, sono abbastanza rappresentativi. Non si può fare a meno di notare che sempre l'energia associata alla scossa principale supera di gran lunga la somma delle energie erogate prima e dopo. A rendere ancora più evidenti tali osservazioni abbiamo calcolato le percentuali delle energie competenti alle tre fasi fondamentali del periodo:

I) Energia sviluppata in tutto il periodo precedente la scossa principale;

II) Energia associata alla scossa principale;

III) Energia sviluppata in tutto il periodo successivo alla scossa principale;

e i grafici della fig. 32 ne esprimono il senso.

La Tabella A riassume tutti i risultati di queste ricerche. Fra i tanti casi esaminati, considerazioni a parte andrebbero fatte per il periodo sismico del 1703. Anzitutto, trattandosi di uno sconvolgimento così tragicamente disastroso, esso non può far legge su quello che può essere lo svolgimento piuttosto normale di una attività regionale circoscritta, e inoltre i centri viciniori essendosi sollecitati a catena non è stato possibile, come s'è detto altrove, discriminare con certezza le manifestazioni secondarie.

Dalla tabella risulta altresi chiaro che in nessun caso (salvo uno) il periodo si è aperto con la scossa principale: in genere questa fa seguito 
ad una attività più moderata che puó incominciare a manifestarsi anche mesi prima. Il caso che fa eccezione è quello del sett.-ott. 1878 con epicentro presso le Fonti del Clitunno: apparentemente il periodo si apre con la scossa principale (quella del 15 sett.) peró, a nostro avviso, riteniamo lecito supporre che qualora si fosse potuto disporre di documentazioni strumentali rilevate nelle vicinanze, anche in questo caso si sarebbero riscontrati dei movimenti preliminari, sia pure di modeste proporzioni.

Nella III parte di questo lavoro avremo modo, disponendo di una ricea documentazione, di esporre i risultati dell'analisi dettagliata del periodo sismico che ha interessato le Sorgenti del Peschiera a partire dal giugno $1961\left(^{*}\right)$.

Prima di chindere la presente nota desideriamo ringraziare il geom. Arcangeli Davide il quale ci ha aiutato nella esecuzione dei disegni.

\section{BIBIJOGRAFIA}

Marceldi I., Pannocchia G., Liatliviti sismica nella zona delle Sorgenti del Peschiera (I parte). "Annali di Geofisica ", 4, (1962).

Barat'Ta II., I terremoti ditalia. Ed. Fratelli Bocea, Torino, 1901.

Cavasivo A., I terremoli d'llalia nel trenacinquennio: 1899-1933. Istituto Poligrafico dello stato, Roma, 1935.

Caror P., Alivilì sismica in Italia nel decennio 1930-39. Reale Accademia d'Italia, Le IIomnier, Firenze.

De Panfuns M., Allivilà sismica in Ilalia dal 19.53 al 1957. "Amnali di Geofisiea ", 1, (1959).

Marcelat L., Monteccin P., Contributi per uno sludio sulla sismicilì dell'llalia. "Annali di Geofisica ", 2, 3, (1962).

Di Filippo D., Marcela L., Uno studio sul terremolo del Gran Sasso d'llalia del 5-9-1950. "Annali di Geofisica ", 2, (1951).

Istituto Nazionale di Geofisica - Roma - marzo 1963.

(*) Per le figure vedere in fondo al volume. 


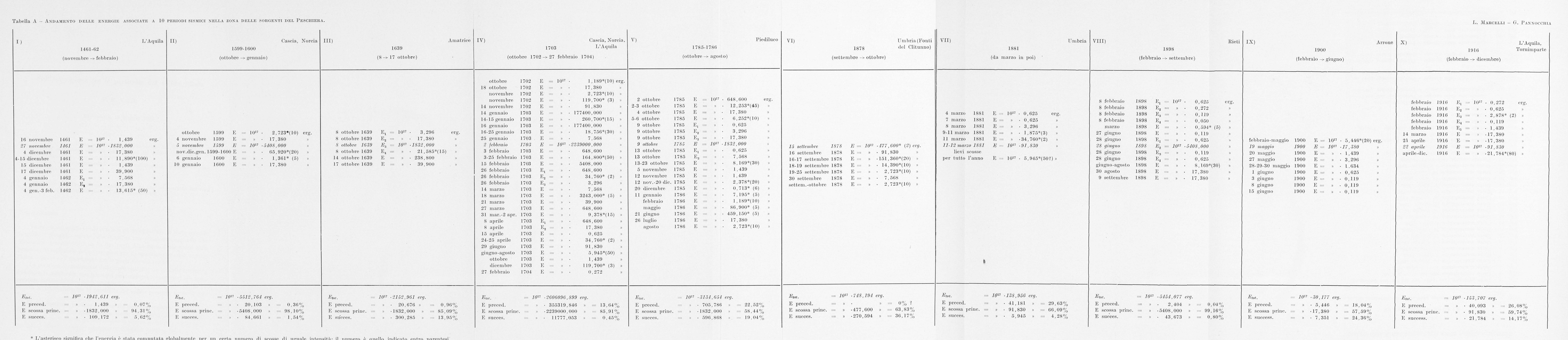

